\title{
A microfluidic system to study the cytotoxic effect of drugs: the combined effect of celecoxib and 5-fluorouracil on normal and cancer cells
}

\author{
Elzbieta Jastrzebska • Sylwia Flis • Aleksandra Rakowska • Michal Chudy • \\ Zenon Jastrzebski • Artur Dybko • Zbigniew Brzozka
}

Received: 18 February 2013 / Accepted: 6 May 2013 /Published online: 21 May 2013

(C) The Author(s) 2013. This article is published with open access at Springerlink.com

\begin{abstract}
We have investigated the response of normal and cancer cells to exposure a combination of celecoxib (Celbx) and 5-fluorouracil (5-FU) using a lab-on-a-chip microfluidic device. Specifically, we have tested the cytotoxic effect of Celbx on normal mouse embryo cells (Balb/c 3T3) and human lung carcinoma cells (A549). The single drugs or their combinations were adjusted to five different concentrations using a concentration gradient generator (CGG) in a single step. The results suggest that Celbx can enhanced the anticancer activity of 5-FU by stronger inhibition of cancer cell growth. We also show that the A549 cancer cells are more sensitive to Celbx than the Balb/c $3 \mathrm{~T} 3$ normal cells. The results obtained with the microfluidic system were compared to those obtained with a macroscale in vitro cell culture method. In our opinion, the microfluidic system represents a unique approach for an evaluation of cellular response to multidrug exposure that also is more simple than respective microwell plate assays.
\end{abstract}

Keywords Chemotherapy resistance $\cdot$ Multidrug tests · Microfluidic gradient system $\cdot$ Lab-on-a-chip · Celecoxib · 5-fluorouracil

Electronic supplementary material The online version of this article (doi:10.1007/s00604-013-1009-4) contains supplementary material, which is available to authorized users.

E. Jastrzebska $(\bowtie) \cdot$ A. Rakowska $\cdot$ M. Chudy $\cdot$ A. Dybko

Z. Brzozka

Department of Microbioanalytics, Institute of Biotechnology,

Warsaw University of Technology, 00-664 Warsaw, Poland

e-mail: ejastrzebska@ch.pw.edu.pl

S. Flis $\cdot$ Z. Jastrzebski

Department of Pharmacology, National Medicines Institute, 00-725 Warsaw, Poland

\section{Introduction}

Cancer therapies have changed dramatically during the past century. Even if surgery or radiotherapy are still used as the primary treatments of many types of cancer, chemotherapy plays also an important role to prevent its recurrence $[1,2]$. In fact, chemotherapeutic drugs are most effective when given in combination (combined chemotherapy). In the combined chemotherapy drugs with different mechanisms of action are used, decreasing the possibility that resistant cancer cells will grow up. When drugs with different effects are combined, each drug can be used at its optimal dose, without intolerable side effects [3]. New anticancer agents are also being tested as well as new combinations tried. Unfortunately, in many cases an objective response rate for treatment is still not satisfactory. There are multiple reasons for chemotherapy failure in patients' treatment, involving a variety of anatomic, pharmacological and biochemical mechanisms. Therefore, instead of unification of cancer treatment for all patients the future is in the personalization of cancer therapy. This generates the needs for adequate assay scheme to efficiently screen new potential drug candidates or/and new drug combinations.

Nowadays, numerous studies demonstrate that the anticancer activity of standard chemotherapeutic agents can be enhanced by using inhibitors of cyclooxygenase-2 (COX-2) enzyme [4]. Nonsteroidal anti-inflammatory drugs (NSAIDs) i.e. celecoxib (Celbx) in combination with cytostatic drug can improve their antitumor effects [5].

In order to better predict the clinical response to drugs' combination, a cell culture model that mimic in vivo behaviour is required [6]. For these purposes, the use of a microfluidic-based cell-culture platform seems to be a promising alternative to the conventional cell culture methods [7, 8]. The construction of small-scale devices permits also for 
using of low cell numbers. It can be important especially during anticancer drug screening of cancer cells isolated from patient. Moreover, development of lab-on-a-chip systems provides novel methods of mimicking the complexity of in vivo conditions through construction of properly defined microchannels geometry. It enables to create high value of the surface-to-volume-ratio (SAV). High value of SAV in live organisms was also observed, therefore suitable designing of this parameter in microchips is important. Besides, lab-on-a-chip devices allow for more accurate modelling of physical situations for both fundamental research and drug development. In specially designed microdevices, the control of essential signals in cellular microenvironments is more precise than in a macroscale $[9,10]$. The cells in organisms are exposed to the microenvironmental signals (i.e. soluble factors, cell-cell interactions, cell-extracellular matrix (ECM), physical forces), which result in activation of cells behaviour. Cellular responses (i.e. self-renewal, differentiation, biosynthesis, metabolism, apoptosis, migration or quiescence) are observed in live tissues [9]. Usually, examination of these bioprocesses in macroscale (on the 96-well plates) is difficult. However, numerous publications described microtools for simultaneous and accurate controlling of various factors such as: cells differentiation, migration and apoptosis [11-13].

Microsystems are used for high-throughput screening after cells exposition with a few signals, including chemical, biochemical, physical and mechanical factors [9, 11-13]. Automatic concentration gradient generators as well as controlled-diffusive mixing play an essential role especially during the cytotoxicity assays [14]. First of all, it allows for real-time monitoring of multifunction conditions/factors in a single plate. Numerous protocols of the toxic effect analysis have been established in miniaturized platforms/systems integrated with a mixing gradient $[12,15-18]$. Irimia et al. described the microdevice developed in PDMS, which contained two completely separated chemical gradients. They were connected through a system of valves to one main channel and two waste channels. The advantage of this type of gradient was a possibility to manipulate a flow, which could be directed either to the main channel or to the waste channel [16]. Evaluation of many independent conditions on various long-term cultures was performed for example by Gomez et al. [19] and Prokop et al. [20]. Cells chemotactic responses, after exposing to different chemokins, were evaluated on neutrophils, breast cancer cells, human neural stem cells or lung carcinoma cells [21-26]. In turn, Hosokawa et al. [27] presented a microfluidic device with chemical gradient for a single-cell cytotoxicity assay. The geometry of this microsystem consists also a microcavity array for entrapment of single cells. In special cavities (chambers) the single Hela cells were trapped and treated with six different concentrations of model compound-potassium cyanine $(\mathrm{KCN})$. Results, presented in this paper, indicated that the microsystem could be used for detection of the concentration- and timedependent cytotoxicity at the single-cell level.

This work presents data of applying microfluidic cell culture system containing concentration gradient generator (CGG) as a platform for evaluation of drugs' combinations. The type of concentration gradient allows generating five different concentrations of two chemotherapeutic agents. We established the cytotoxic effect of Celbx and 5-FU on normal mouse embryo cells (Balb/c 3T3) and human lung carcinoma cells (A549). For this purpose we used an integrated microfluidic gradient system, which can be used as an alternative tool for rapid multidrug exposure examination [28]. Moreover, response of both tested cell lines treated with the combination of Celbx and 5-FU was examined. These studies allowed for investigation of anticancer properties of Celbx.

\section{Experimental}

\section{Biological materials}

The A549 (human lung carcinoma cell line) and Balb/c 3T3 (normal mouse embryo cell line) obtained from the ATCC collection (Manassas, VA, USA, www.lgcstandardsatcc.org) were used for cytotoxicity assays in both microand macroscale experiments. Both macro- and microscale cell cultures were carried out using the same media and regime of medium exchange [28]. A549 cells were chosen because lung cancer is one of the most common cancers in the world as well as leading cause of cancer death in men and women. Therefore, it gets an important social problem. In turn, Balb/c 3T3 cell line, was chosen because the European Centre of Validation of Alternative Methods (ECVAM) considered this in vitro model system for evaluating the cytotoxicity and morphological transformation of chemicals and metal compounds [29]. Moreover, Balb/c 3T3 cells represent normal tissue, what seems to be a good model for analysis of anticancer activity of the chosen drugs.

\section{Assays in a microsystem}

The reusable, hybrid (PDMS/glass) microsystem was used during the studies (Fig. 1). Design and method of fabrication of the microfluidic cell culture system for cytotoxicity tests was presented in details in our previous work [30]. The inhibitory effect of combination of two drugs (Celbx and 5 -FU) was analyzed on the cells introduced and cultured in the microsystem (see ESM). However, before these studies the cytotoxic effect of single drug was investigated. Therefore, 
Fig. 1 a The microfluidic system integrated with concentration gradient generator (CGG) consists of PDMS and glass. b Microchambers with Balb/c $3 \mathrm{~T} 3$ and A549 cells cultured for $24 \mathrm{~h}$

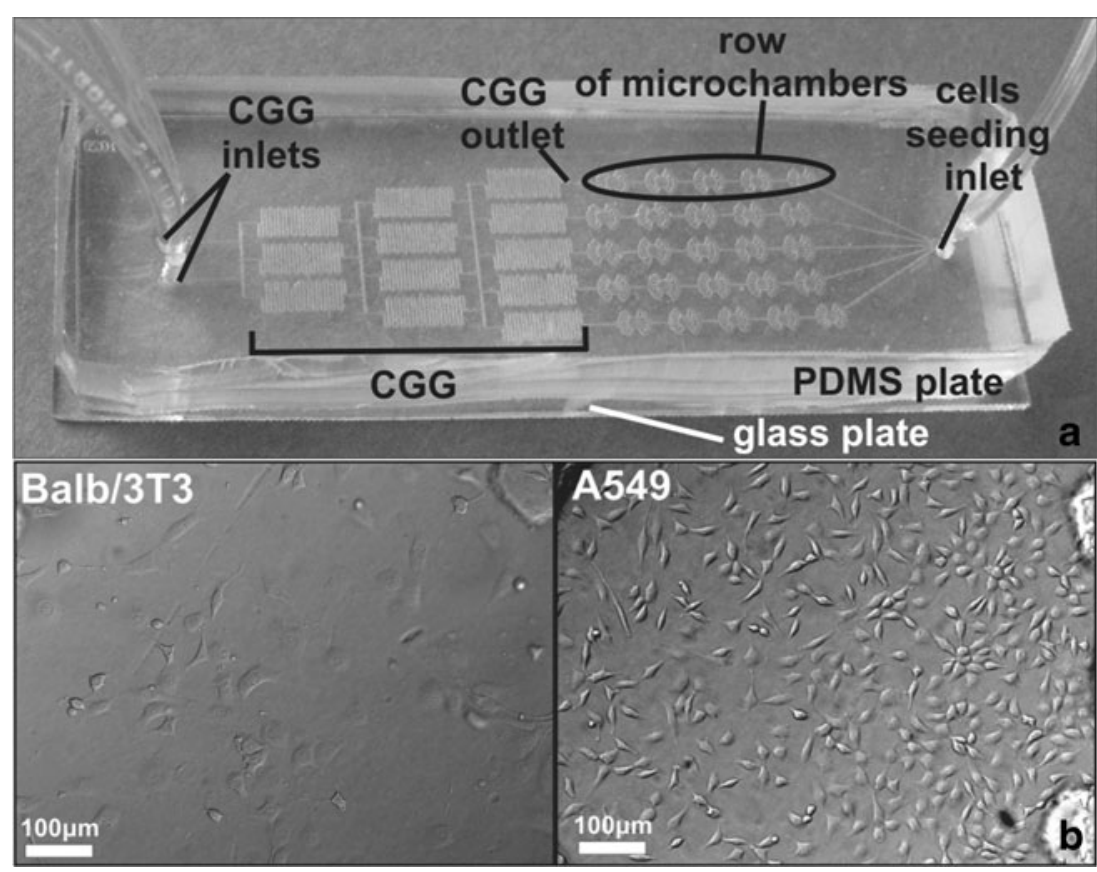

both the A549 and Balb/c 3 T3 cells response on five various concentration of Celbx (range of $0 \div 120 \mu \mathrm{M}$ ) and 5-FU (range of $0 \div 300 \mu \mathrm{M}$ ) was established in separate tests. After mixing in $\mathrm{CGG}$, in consecutive rows of culture microchambers, five different concentrations of single drug was obtained. After evaluation of the cytotoxic effect of single treatment, multidrug tests in the microsystem were performed. In this purpose, two different solutions of Celbx $(120 \mu \mathrm{M})$ and 5-FU $(300 \mu \mathrm{M})$ into the microsystems were pumped. Drugs were introduced through the CGG inlets with a flow rate of $1.2 \mu \mathrm{L} \mathrm{min}{ }^{-1}$ for $50 \mathrm{~min}$. The microdevices with the introduced drugs (in both single and multidrug treatment) were placed in the incubator for the next 24 or $48 \mathrm{~h}$. For each tested drug, the separate microsystem with proper cell line was used. Finally, cell viability assay was performed using fluorescent dyes (see ESM).

\section{Assays in a macrosystem}

Both cell lines (at a density of $1 \times 10^{4}$ cells per well) were placed into flat bottom 96-well plates and allowed to attach overnight. Growth inhibitory effects of Celbx and 5-FU were evaluated by the MTT (3-(4,5dimethylthiazol-2-yl)-2,5-diphenyltetrazolium bromide) assay (www.invitrogen.com), which measures mitochondrial activity of viable cells. The cells were simultaneously treated with Celbx and 5-FU or with each drug separately for 24 and $48 \mathrm{~h}$. After the treatment procedure, the medium was removed and cells were incubated for $4 \mathrm{~h}$ at $37^{\circ} \mathrm{C}$ in $50 \mu \mathrm{L}$ per well with MTT solution (final concentration $0.5 \mathrm{mg} \mathrm{mL}^{-1}$ ). Formazan crystals were dissolved in $150 \mu \mathrm{L}$ of $10 \%$ SDS solution per well and the absorbance was measured at $540 \mathrm{~nm}$ using a spectrophotometric microplate reader Power Wave XS (Bio-Tek, Winooski, VT, USA).

\section{Statistical analysis}

Experimental data are expressed as mean \pm standard deviation (SD) from at least four independent experiments. Data were analysed using SigmaPlot version 11 software. Statistical analysis was performed using the one-way ANOVA. Values of $P<0.05$ were considered to be statistically significant.

\section{Results and discussion}

\section{Cytotoxicity assay}

Good coherence between the theoretical and experimental data during a flow of solution through the microdevice, particularly in the CGG has been proved [28]. Results on microsystem operations are given in ESM. The geometry of the fabricated microsystem achieved uniform distribution of cells in microchambers. Analysis within $24 \mathrm{~h}$ (using a microscope) of introduced A549 and Balb/c 3T3 cells showed good morphology, comparable to control experiments in which cells were cultured in 96-well plates. Figure $1 \mathrm{~b}$ shows A549 and Balb/c 3T3 cells cultured in the microchambers of the microsystem for $24 \mathrm{~h}$. Good adhesion to the glass surface was observed. Two cell lines selected for the investigation represent normal and cancer tissue, what seems to be a good model for analysis of anticancer activity of the chosen drugs, i.e. Celbx and 5-FU. The microsystem 
enabled to analyse of cell morphology and cytotoxicity simultaneously.

Both cultured cell lines were exposured to combination of Celbx with 5-FU. Additionaly, for the comparision the cytotoxic effect of single drug was also evaluated. In our previous work, we described results for incubation of A549 cells with 5-FU [28]. Here, we investigated the cytotoxic effect of Celbx on A549 and Balb/c 3T3 cells as well as 5$\mathrm{FU}$ on Balb/c 3T3 cells (see ESM). Moreover, the cytotoxic effect on the cells after multidrug exposure was investigated.

Both cell lines were simultaneously exposed to Celbx ( 0 $120 \mu \mathrm{M})$ and 5-FU $(0-300 \mu \mathrm{M})$ in the microfluidic device (microsystem). Cells were exposed to different concentration for 24 and $48 \mathrm{~h}$. Figure 2 shows that Celbx and 5-FU, separately dosed, exert time-dependent cytotoxic effect on carcinoma A549 cells. The strongest cytotoxic effect of single drug was determined for concentrations from 60 to $120 \mu \mathrm{M}$ for Celbx and from 150 to $300 \mu \mathrm{M}$ for 5 -FU. Incubation of the cells with combination of tested drugs enhanced the cytotoxic effect in comparison to drugs introduced separetly. For each period of time ( 24 and 48 h) viability decrease of A549 cells' after incubtion with combination of Celbx and 5-FU was observed. The ranges of drugs' concentrations, in which enhanced cytotoxic effect is the strongest can be determined (Fig. 2). Decrease of A549 cells' viability was observed in ranges of Celbx $(39 \div$
$83 \mu \mathrm{M})$ and 5 -FU $(202 \div 93 \mu \mathrm{M})$ for $24 \mathrm{~h}$ and Celbx $(19 \div$ $117 \mu \mathrm{M})$ and 5 -FU $(253 \div 8 \mu \mathrm{M})$ for $48 \mathrm{~h}$. The lowest A549 cells' viability ( $30 \%$ ) after $48 \mathrm{~h}$ incubation with combination of $60 \mu \mathrm{M}$ Celbx and $150 \mu \mathrm{M} 5$-FU in comparison to single drugs administration $(50 \%)$ was determined.

Similar experiments were performed on Balb/c 3T3 cells. Figure 3 shows that both agents as well as their combinations were less effective on Balb/c 3T3 cells. Exposure to Celbx (without 5-FU), which seems to be the most potent agent, decrease to $65 \% \mathrm{Balb} / \mathrm{c} 3 \mathrm{~T} 3$ cell viability at the highest concentration tested after 24 and $48 \mathrm{~h}$ of incubation. Whereas, in the same conditions $(120 \mu \mathrm{M}$ of Celbx $)$, the viability of the A549 cells decreased to $20 \%$. The results obtained in this study suggest that Celbx can enhance anticancer activity of 5-FU by stronger inhibition of cancer cell growth as well as indicate its own anticancer activity. However, we observed that application of both drugs enhanced also the cytotoxic effect of the Balb/c 3T3 cells. Balb/c 3T3 cell viability decreased after incubation with all drugs' combination.

Comparison the toxic effect of the tested drugs between micro and macroscale

The results obtained with the microfluidic device were compared with those obtained using conventional in vitro cell
Fig. 2 Effects of celecoxib (Celbx), 5-fluorouracil (5-FU) or their combination on viability of A549 in microfluidic system and 96well plates after 24 and $48 \mathrm{~h}$. Data shown presents averages of four independent experiments. Asterisks indicate $P<0.05$

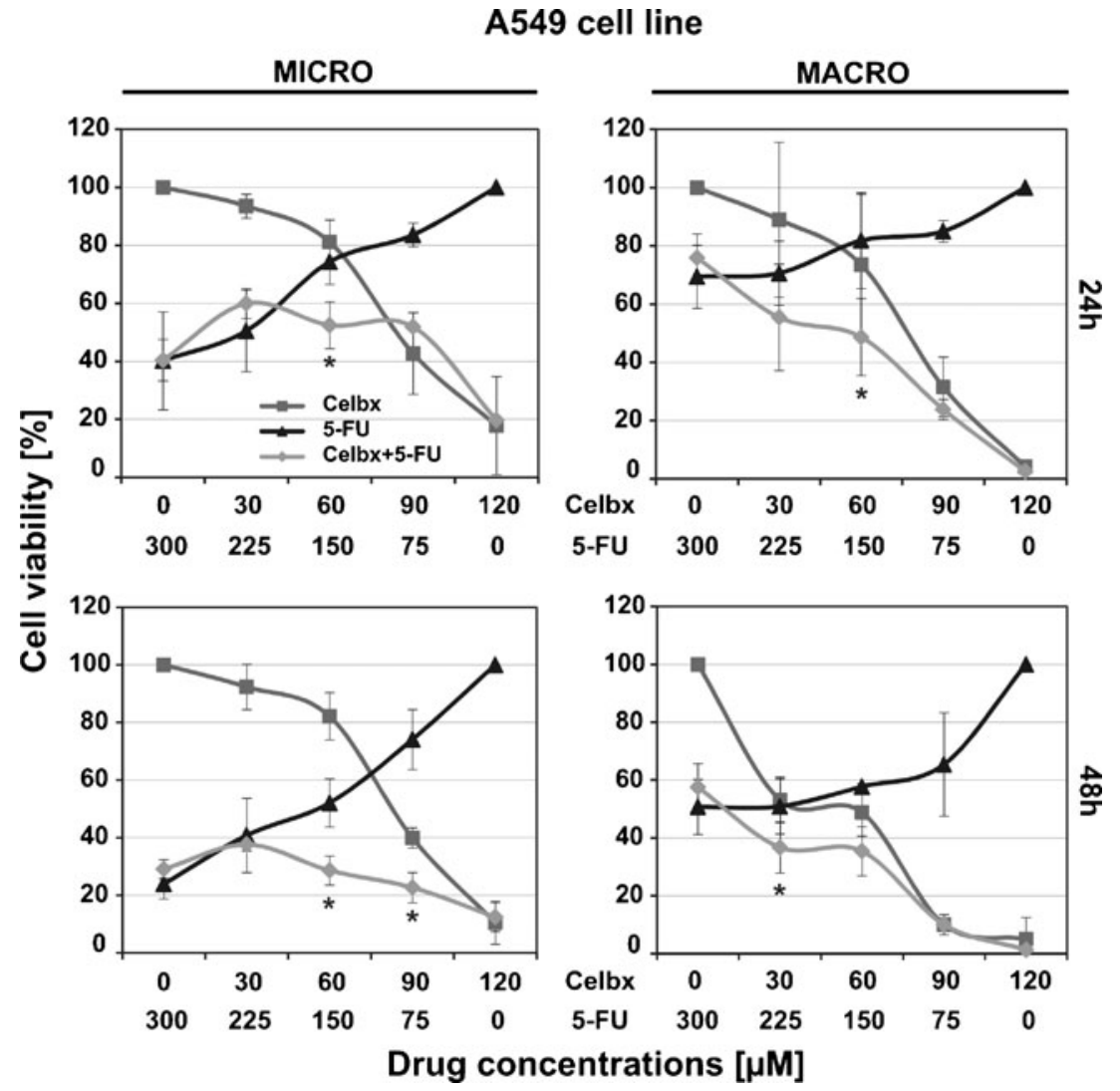


Fig. 3 Effects of celecoxib (Celbx), 5-fluorouracil (5-FU) or their combination on viability of Balb/c $3 \mathrm{~T} 3$ cells in microfluidic system and 96well plates after 24 and $48 \mathrm{~h}$. Data shown presents averages of four independent experiments. Asterisks indicate $P<0.05$

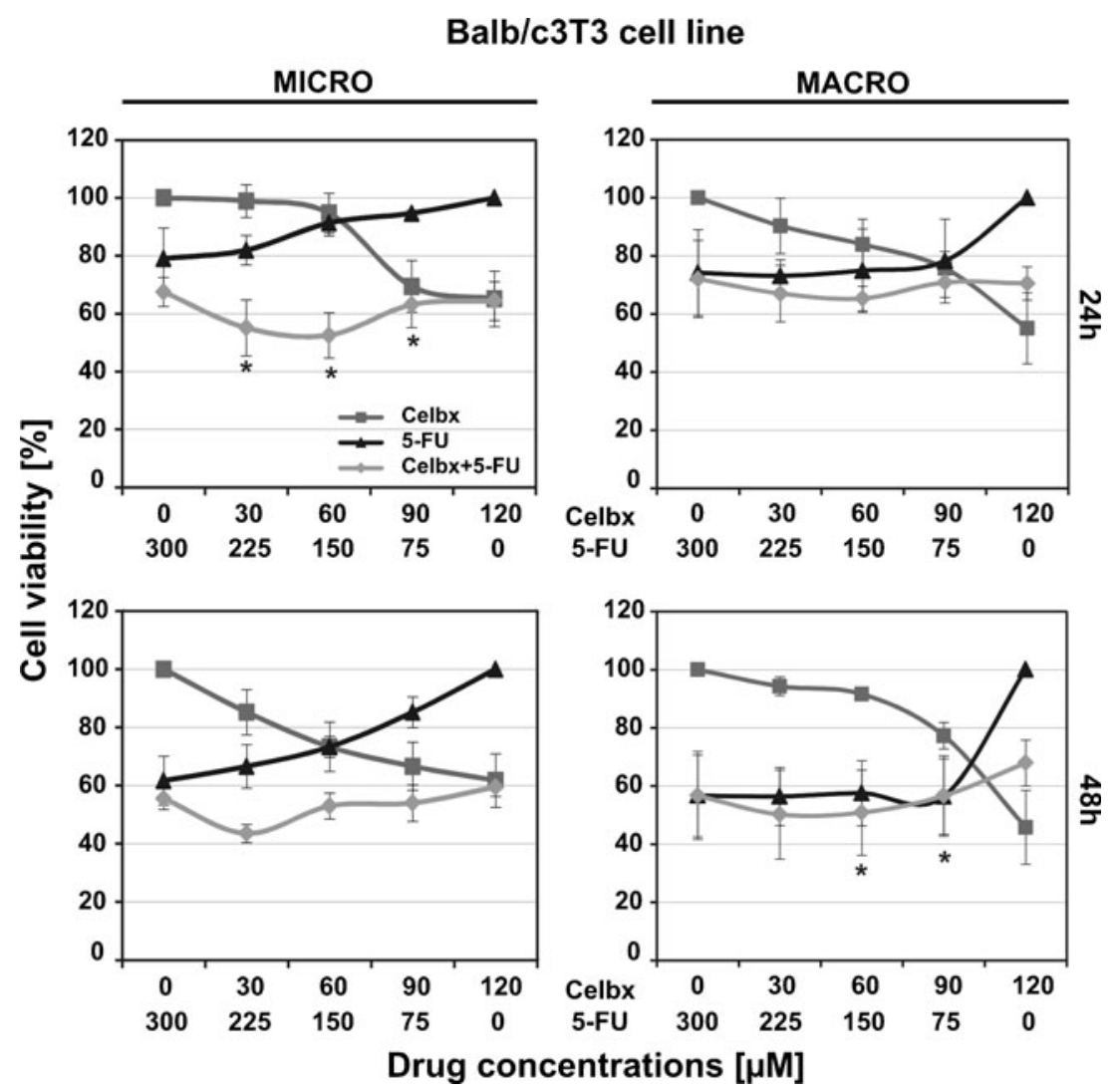

culture method (macrosystem) in a 96-well plate at selected concentrations (Figs. 2 and 3). The presented results show that cell treatment in the microfluidic device lead to a smaller decrease of viability of A549 cells than in 96-well plates. The enhanced cytotoxic effect for both cell lines in micro- and macroscale was observed. The highest discrepancy between both scales for A549 cells $24 \mathrm{~h}$ incubated with drugs' combination was established. Our results obtained for single drug treatment in both cultivation types (microsystem and 96-well plate) are more comparable than those obtained for drug combination treatment. Regression analysis indicates significant correlation of Celbx and 5-FU cytotoxicity between two evaluated systems, because high Pearson's correlation coefficients (R2) were calculated. For single treatments, in most cases, we obtained positive correlation coefficient parameters with $\mathrm{P}$ values below 0.05 (Table 1).

Moreover, 5-FU at the highest tested concentration is stronger in the microsystem than in the 96-well plate. Additionally, Balb/c 3T3 cells were more sensitive to drug combinations in the microsystem (Fig. 3).

Several factors could have influence on these discrepancies in drug efficiency between the evaluated systems. Most likely, the discrepancy between the two evaluated systems could result from the differences in the cell culture conditions and different access of the anticancer drug to the cells cultivated in the macroscale. First of all, in the 96-well plates, cells were cultured in static conditions, while in the microsystem culture media were periodically replaced and cell cultures were rinsed with drugs and solution of CAM and PI. However, the flow of reagents that occurs in our microdevice, can mimic in vivo condition during the provision of drugs and substances into the living body. On the other hand, the cells division in microscale (where SAV is high [10])

Table 1 The Pearson correlation values (R) between microfluidic device (microsystem) and well plate culture (macrosystem) calculated for single treatments

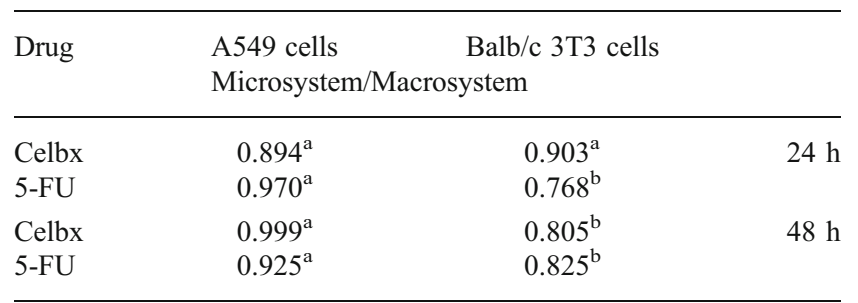

${ }^{\mathrm{a}}$ The pair of variables with positive correlation coefficients and $\mathrm{P}$ value below 0.05 tends to increase together

${ }^{\mathrm{b}}$ For pairs with $\mathrm{P}$ values greater than 0.05 , there is no significant relationship between the two variables 
can result from differences in the cells response on the drugs exposure.

Another hypothesis concerning the obtained discrepancy could result from absorption in PDMS of the tested cytotoxic drugs. Therefore, we investigated absorption of drugs, according to previously elaborated procedure utilizing gas chromatography $[28,30]$. We proved that after several tests performed in the same microsystem, the PDMS block did not absorb the 5-FU (data shown in previous publication) as well as Celbx. In the chromatogram of standard solution of Celbx, there were peaks corresponding to Celbx (retention time $16.47 \mathrm{~min}$ ) and DMSO (retention time $1.33 \mathrm{~min}$ ). We investigated that the tested PDMS pieces (obtained from the microsystem) contains only DMSO solution (retention time $1.33 \mathrm{~min}$ ). We proved that in this case differences between micro and macro scale can not result from absorption in PDMS of the tested cytotoxic drugs. However, the usage of other drugs requires examination of their absorption in PDMS.

\section{Conclusions}

This study demonstrated response of human lung carcinoma cells (A549) and normal mouse embryo cells (Balb/c 3T3) cells on the exposure of combination celecoxib (Celbx) with 5-fluorouracil (5-FU). The application of the CGG in the microsystem improved repeatable generation of different drugs concentration. Moreover, during the multidrug studies different mixtures of the tested drugs (Celbx and 5-FU) were obtained automatically and simultaneously. It allows avoiding operator's errors, which can occur during the preparation of the solutions and it enables to obtain reproducible results. The calculated correlations and high values of Pearson parameter between micro- and macroscale have proved that the microsystem can be an alternative tool for performing the multidrug cytotoxicity assays. The results suggest that Celbx, nonsteroidal anti-inflammatory drug (NSAID), inhibits the growth of cancer cells and indicates anticancer properties. We proved that after cells incubation with Celbx the viability of A549 cells was lower than Balb/c 3T3 normal cells. Moreover, Celbx in combination with 5-FU enhances the antitumor activity. The results confirmed that Celbx inhibits the growth of cancer cells as well as indicates its anticancer properties. The results obtained in the microfluidic system were also compared with classical tests (macroscale). Our results indicate that the microfluidic device can be used for testing the cytotoxic effect of drugs' combination. It is important to note that despite of differences between both evaluated systems, the microfluidic device shows the possibility to test the cytotoxic effect of both drugs: Celbx and 5-FU. This comparison (micro with macroscale) proved possibility to perform multidrug tests in microscale. Our results suggest that the fabricated microfluidic system (containing CGG) can be a unique approach for first evaluation of the cellular responses. It gives possibility for development of simplified methods for simultaneous testing of anticancer drugs or other new synthesized compounds, which are potentially biological active. We believe that our investigations improve validation and standardization of microsystem for cytotoxicity assays.

Acknowledgments The authors would like to thank Renata Krzysztofik from the Department of Pharmacology of National Medicines Institute for MTT measurement. The authors would like to thank Anna Jerzak from the Laboratory of Technological Processes (WUT) for $\mathrm{GC}$ measurement. This work was financially supported within a frame of project number 1450/B/H03/2011/40 financed by the Nation Centre of Research and LIDER program No LIDER/17/202/L-1/09/NCBiR/2010

Open Access This article is distributed under the terms of the Creative Commons Attribution License which permits any use, distribution, and reproduction in any medium, provided the original author(s) and the source are credited.

\section{References}

1. Danesi DT (2010) The association of chemotherapy and radiotherapy: biological rationale. Curr Drug The 5:152

2. Gadgeel SM, Ramalingam SS, Kalemkerian GP (2012) Treatment of lung cancer. Radiol Clin N Am 5:961

3. Ullah MF (2008) Cancer Multidrug Resistance (MDR): a major impediment to effective chemotherapy. Asian Pac J Cancer Prev 9:1

4. Irie T, Tsujii M, Tsuji S, Yoshio T, Ishii S, Shinzaki S, Egawa S, Kakiuchi Y, Nishida T, Yasumaru M, Iijima H, Murata H, Takehara T, Kawano S, Hayashi N (2007) Synergic antitumor effects of celecoxib with 5-fluorouracil depend on INF-y. Int J Cancer 4:878

5. Wilgus TA, Breza TS, Tober KL, Oberyszyn TM (2004) Treatment with 5-fluorouracil and celecoxib displays synergistic regression of ultraviolet light B-induced skin tumors. J Investig Dermatol 122:1488

6. Wu MH, Huang SB, Lee GB (2010) Microfluidic cell culture systems for drug research. Lab Chip 10:939

7. Yeo LY, Chang HC, Chan PP, Friend JR (2011) Microfluidic devices for bioapplications. Small 7:12

8. Noort D, Ong SM, Zhang C, Arooz T, Yu H (2009) Stem cells in microfluidics. Biotechnol Prog 25:52

9. Gupta K, Kim DH, Ellison D, Smith C, Kundu A, Suh KY, Tuan J, Levchenko A (2010) Lab-on-a-chip devices as an emerging platform for stem cell biology. Lab Chip 10:2019

10. Beebe DJ, Mensing GA, Walker GM (2002) Physics and applications of microfluidics in biology. Annu Rev Biomed Eng 4:261

11. Neužil P, Giselbrecht S, Länge K, Huang TJ, Manz A (2012) Revisiting lab-on-a-chip technology for drug discovery. Nat Rev Drug Discov 11:620

12. Hung PJ, Lee PJ, Sabounchi P, Aghdam N, Lin R, Lee LP (2005) A novel high aspect ratio microfluidic design to provide a stable and uniform microenvironment for cell growth in a high throughput mammalian cell culture array. Lab Chip 5:44

13. Kim DH, Wong PK, Park J, Levchenko A, Sun Y (2009) Microengineered platforms for cell mechanobiolog. Annu Rev Biomed Eng 11:203

14. Dertinger KW, Chiu DT, Jeon NL, Whitesides GM (2001) Generation of gradients having complex shapes using microfluidic networks. Anal Chem 73:1240 
15. Chung BG, Lin F, Jeon NL (2006) A microfluidic multi-injector for gradient generation. Lab Chip 6:764

16. Irimia D, Liu SY, Tharp WG, Samadani A, Toner M, Poznansky MC (2006) Microfluidic system for measuring neutrophil migratory responses to fast switches of chemical gradients. Lab Chip 6:191

17. Fung WT, Beyzavi A, Abgrall P, Nguyen NT, Li HY (2009) Microfluidic platform for controlling the differentiation of embryoid bodies. Lab Chip 9:2591

18. Piraino F, Camci-Unal G, Hancock MJ, Rasponi M, Khademhosseini A (2012) Multi-gradient hydrogels produced layer by layer with capillary flow and crosslinking in open microchannels. Lab Chip 12:659

19. Gomez-Sjoberg R, Leyrat AA, Pirone DM, Chen CS, Quake SR (2007) Versatile, fully automated, microfluidic cell culture system. Anal Chem 79:8557

20. Prokop A, Prokop Z, Schaffer D, Kozlov E, Wikswo J, Cliffel D, Baudenbacher F (2004) NanoLiterBioReactor: monitoring of long-term mammalian cell physiology at nanofabricated scale. Biomed Microdevices 6:325

21. Jeon N, Baskaran H, Dertinger SK (2002) Neutrophil chemotaxis in linear and complex gradients of interleukin-8 formed in a microfabricated device. Nat Biotechnol 20:826

22. Wang SJ, Saadi W, Lin F, Nguyen CMC, Jeon NL (2004) Differential effects of EGF gradient profiles on MDA-MB-231 breast cancer cell chemotaxis. Exp Cell Res 300:180
23. Chung BG, Flangan LA, Rhee SW, Schwartz PH, Lee AP, Monuki ES, Jeon NL (2005) Human neural stem cell growth and differentiation in a gradient-generating microfluidic device. Lab Chip 5:401

24. Siyan W, Feng Y, Lichuan Z, Jiarui W, Yingyan W, Li J, Bingcheng L, Qi W (2009) Application of microfluidic gradient chip in the analysis of lung cancer chemotherapy resistance. J Pharm Biomed Anal 49:806

25. Dertinger S, Jiang X, Li Z, Murthy V, Whitesides GM (2002) Gradients of substrate-bound laminin orient axonal specification of neurons. PNAS 99:12542

26. Ma B, Zhang GH, Qin JH, Lin BC (2009) Characterization of drug metabolites and cytotoxicity assay simultaneously using an integrated microfluidic device. Lab Chip 9:232

27. Hosokawa M, Hayashi T, Mori T, Yoshino T, Nakasono S, Matsunaga T (2011) Microfluidic device with chemical gradient for single-cell cytotoxicity assays. Anal Chem 83:3648

28. Jedrych E, Flis S, Sofinska K, Jastrzebski Z, Chudy M, Brzozka Z (2011) Evaluation of cytotoxic effects of 5-fluorourcil on human carcinoma cells in microfluidic system. Sensors Actuators B Chem 160:1544

29. Corvi R, Aardema MJ, Gribaldo L, Hayashi M, Hoffmann S, Schechtman L, Vanparys P (2012) ECVAM prevalidation study on in vitro cell transformation assays: general outline and conclusions of the study. Mutat Res 11:12

30. Ziolkowska K, Jedrych E, Kwapiszewski R, Lopacinska J, Skolimowski M, Chudy M (2010) PDMS/glass Microfluidic cell culture system for cytotoxicity tests and cells passage. Sensors Actuators B Chem 145:533 